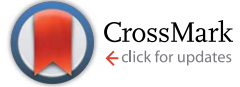

Cite this: J. Mater. Chem. A, 2016, 4, 7555

Received 1st March 2016 Accepted 1st April 2016

DOI: $10.1039 / c 6 t a 01828 a$

www.rsc.org/MaterialsA

\section{Hollow nanoporous covalent triazine frameworks via acid vapor-assisted solid phase synthesis for enhanced visible light photoactivity $\dagger$}

\author{
Wei Huang, ${ }^{a}$ Zi Jun Wang, ${ }^{a}$ Beatriz Chiyin Ma, ${ }^{a}$ Saman Ghasimi, ${ }^{a}$ Dominik Gehrig, ${ }^{a}$ \\ Frédéric Laquai, ${ }^{b}$ Katharina Landfester ${ }^{a}$ and Kai A. I. Zhang*a
}

Herein, we report a novel trifluoromethanesulfonic acid vapor-assisted solid phase synthetic method to construct nanoporous covalent triazine frameworks with highly ordered hollow interconnected pores under mild reaction conditions. This unique solid state synthetic route allows not only the avoidance of undesired side reactions caused by traditional high temperature synthesis, but also the maintaining of defined and precise optical and electronic properties of the nonporous triazine frameworks. Promising photocatalytic activity of the polytriazine networks was demonstrated in the photoreduction reaction of 4-nitrophenol into 4-aminophenol under visible light irradiation.

Covalent triazine frameworks (CTFs), a type of nitrogen-rich porous polymer sharing similar triazine building blocks as in carbon nitrides, have been usually prepared via trimerization reactions of aromatic nitriles in molten $\mathrm{ZnCl}_{2}$ at high temperature (400-700 $\left.{ }^{\circ} \mathrm{C}\right) .{ }^{1}$ They have demonstrated excellent performance in various application fields, such as energy storage, ${ }^{2-5}$ catalysis, ${ }^{6-9}$ and gas absorption/separation, ${ }^{\mathbf{1 0 - 1 3}}$ due to their extraordinary chemical stability and high porosity. However, the optical property of CTFs has been largely ignored. Recent theoretical simulations revealed their semiconductor character with broad absorption in visible range and predicted the potential of CTFs as visible light-active photocatalysts. ${ }^{14-17}$ To date, little experimental evidence of their photocatalytic property has been reported. ${ }^{\mathbf{1 4 , 1 8 , 1 9}}$ The limited utilization of CTFs in photocatalysis is likely caused by the harsh synthesis condition, which could easily lead to partial carbonization of the polymer, making the conduction band (CB) and valence band (VB)

${ }^{a}$ Max Planck Institute for Polymer Research, Ackermannweg 10, 55128 Mainz, Germany. E-mail: kai.zhang@mpip-mainz.mpg.de

${ }^{b}$ Material Science and Engineering Department, King Abdullah University of Science and Technology (KAUST), Thuwal 23955-6900, Kingdom of Saudi Arabia

$\dagger$ Electronic supplementary information (ESI) available: Detailed experimental procedures, characterization data of BET surface area, PXRD patterns, elemental analysis, solid state NMR, TGA, electrochemical analysis, fluorescence decay spectra and additional SEM and TEM images. See DOI: 10.1039/c6ta01828a positions uncontrollable. The development of milder reaction conditions is therefore of great interest.

In addition to the chemical composition variation, morphology control of heterogeneous photocatalysts has been considered an important tool for optimizing catalytic efficiency. ${ }^{\mathbf{2 0 - 2 2}}$ Among various morphologies, hollow architectures are of particular interest. They have been shown to facilitate mass transfer and increase absorption efficiency by multiple light reflection during the catalytic process and subsequently enhance photocatalytic activity. ${ }^{23}$ The liquid-phase reaction conditions of CTFs, either in molten $\mathrm{ZnCl}_{2}$ or trifluoromethanesulfonic acid $(\mathrm{TfOH})$ solution, are not advantageous to construct highly ordered hollow nanostructures. It is therefore our desire to develop a novel method to prepare CTFs with precious chemical composition coupled with controllable hollow nanostructures for enhanced visible light photocatalysis.

Herein, we report a facile solid phase synthetic method to produce nanoporous CTFs based on trimerization reaction of nitrile-functionalized aromatic units catalyzed by TfOH vapor at elevated temperature $\left(100{ }^{\circ} \mathrm{C}\right)$ in solid phase. To achieve ordered and defined hollow nanostructure, uniformly packed silica nanoparticles ( $\mathrm{SiO}_{2} \mathrm{NPs}$ ) with the size of $c a .300 \mathrm{~nm}$ were employed as removable templates. Enhanced photocatalytic activity of the nanoporous polytriazine networks was demonstrated in the photocatalytic reduction of 4-nitrophenol (4-NP) to 4-aminophenol (4-AP) as a simple test reaction under visible light irradiation. The results suggested that solid vapor synthesis under mild reaction conditions was an efficient preparation method of nanoporous CTFs with defined optical and electronic property and ordered hollow structure. Furthermore, not only the chemical composition but also the morphological structure had a significant effect on the photocatalytic performance of the CTFs.

Scheme 1 illustrates the $\mathrm{TfOH}$ vapor-assisted synthetic method of nanostructured CTFs with interconnected hollow spherical pores. A photoactive and electron donor-acceptor type monomer, 4,4'-(benzothiadiazole-4,7-diyl)dibenzonitrile $\left(\mathrm{BT}-\mathrm{Ph}_{2}-\mathrm{CN}_{2}\right)$, in solid phase mixed with tightly packed $\mathrm{SiO}_{2}$ 


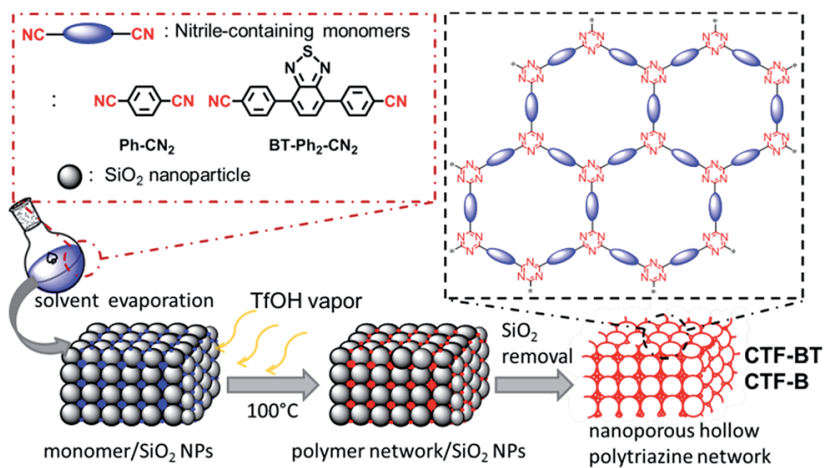

Scheme 1 Schematic of solid vapor synthesis and idealized structures of the nanoporous hollow polytriazine networks.

NPs was polymerized under $\mathrm{TfOH}$ vapor at elevated temperature $\left(100{ }^{\circ} \mathrm{C}\right)$ in a sealed container, obtaining the nanoporous polymer CTF-BT after the removal of the silica templates. Such donor-accepter arrangement would improve the electron delocalization, lower the energy level and thus enhance the visiblelight responsibility. ${ }^{24}$ A known CTF containing only nitrilefunctionalized phenyl unit (CTF-B) with similar hollow structure was synthesized as a comparison to investigate the chemical composition effect on the photocatalytic efficiency of the nanostructured CTFs. Experimental details and characterization data are described in the ESI. $\dagger$

The unique hollow structures of both CTFs were readily identified by the scanning electron microscopy (SEM) and transmission electron microscopy (TEM). As shown in Fig. 1, the images of the CTFs illustrated an interconnected porous structure with a diameter of $c a .300 \mathrm{~nm}$, which corresponds with the diameter of the $\mathrm{SiO}_{2}$ NPs (Fig. S1, ESI $\dagger$ ). The shell thickness of the pores ranged from $30 \mathrm{~nm}$ to $50 \mathrm{~nm}$. No apparent collapse of the macropores was observed after removing the template, suggesting a rigid and robust polymer skeleton. Interestingly, the existence of randomly distributed small holes on the shells was clearly observed (Fig. 1a and c), which could be beneficial for enhanced mass transfer throughout the highly porous polymer network. In comparison, a reference CTF with similar structure as CTF-B was obtained using the same $\mathrm{SiO}_{2}$ template in TfOH solution. It could be observed that only randomly distributed hollow macropores were obtained in the polymer, which were not orderly interconnected due to the absence of tightly stacked silica nanoparticles (Fig. S2†). This demonstrates the advantage of the TfOH vapor-assisted synthesis for the construction of highly ordered hollow nanostructures.

The Brunauer-Emmett-Teller (BET) surface area of CTF-BT was determined to be $c a .90 \mathrm{~m}^{2} \mathrm{~g}^{-1}$. In comparison, the BET surface area of CTF-B was measured to be $565 \mathrm{~m}^{2} \mathrm{~g}^{-1}$ (Fig. S3-S6 and Table S1, ESI $\dagger$ ). It should be addressed that both CTFs showed much higher BET surface areas than that prepared via liquid phase synthesis with usually no porosity. ${ }^{16,25}$ Fourier transform infrared (FTIR) spectra of the polymers showed intense peaks at 1503 and $1350 \mathrm{~cm}^{-1}$, owing to the typical vibrational and stretching modes of aromatic $\mathrm{C}-\mathrm{N}$ bonds, indicating successful formation of triazine units (Fig. 2a). ${ }^{1}$ The signals at $2220 \mathrm{~cm}^{-1}$, which are typical for terminal cyano groups, disappeared or were highly attenuated and no evidence of possible hydrolysis of the nitrile groups was observed. In contrast, the same polymers obtained via liquid synthesis in TfOH solution ${ }^{16}$ showed broad bands between 3700 and $2750 \mathrm{~cm}^{-1}$ and an intense signal at $1650 \mathrm{~cm}^{-1}$, which are characteristic for amide groups formed via hydrolysis of the cyano units (Fig. S7, ESI $\dagger$ ) ${ }^{26}$ This indicates the advantage of the solid/vapor synthesis of nanoporous CTFs by effectively inhibiting the hydrolysis of terminal functional groups. The powder X-ray diffraction (XRD) pattern of CTF-BT showed only a broad diffraction peak at $\sim 25^{\circ}$, suggesting an amorphous character with a disordered network (Fig. S9a, ESI $\uparrow$ ). CTF-B, however, exhibited an intensity diffraction peak at $\sim 7^{\circ}$, which corresponds to the reflection from (100) plane, indicating the existence of a long-range molecular ordering in the polymer network of CTF-B (Fig. S9b, ESI $\dagger$ ).

Solid state ${ }^{13} \mathrm{C} / \mathrm{MAS}$ NMR spectra of the CTFs showed a characteristic signal of the $\mathrm{sp}^{2}$ carbon in the triazine ring at $171 \mathrm{ppm}$ (Fig. S10, ESI $\dagger$ ). The signals between 110 and 150 ppm can be assigned to aromatic carbons in the polymer backbones. No apparent structural decomposition was observed by elemental analysis (Table S2, ESI $†$ ). Thermal gravimetric analysis (TGA) revealed an excellent thermal stability up to $500{ }^{\circ} \mathrm{C}$ under oxygen atmosphere (Fig. S11, ESI $\dagger$ ), which was comparable to CTFs synthesized under high temperature ionothermal conditions.

The UV-vis diffuse reflectance (DR) spectrum of CTF-BT showed a broader absorption band in the visible region up to $550 \mathrm{~nm}$ (Fig. 2b), while CTF-B only absorbed mostly in the UV range $(\lambda<400 \mathrm{~nm})$. Similar behavior of an extended absorption range was demonstrated by introducing the BT units into porous organic polymer networks. ${ }^{27}$ Optical band gaps $\left(E_{\mathrm{g}}\right)$ $2.42 \mathrm{eV}$ for CTF-BT and $3.32 \mathrm{eV}$ for CTF-B could be derived from the Kubelka-Munk-transformed reflectance spectra (Fig. S12, ESI $\dagger$ ). The fluorescence spectra of both CTFs with maxima of $542 \mathrm{~nm}$ for CTF-BT and $427 \mathrm{~nm}$ for CTF-B are observed (Fig. S13, ESI $\dagger$ ), showing similar behavior to the UV-vis absorption spectra of the CTFs.

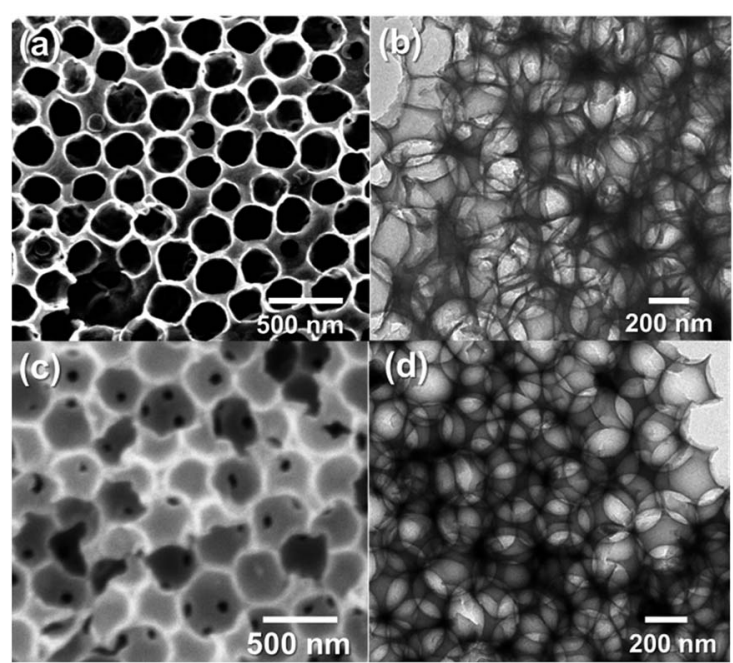

Fig. 1 Typical SEM and TEM images of $(a, b)$ nanoporous hollow polymers CTF-BT and (c, d) CTF-B. 

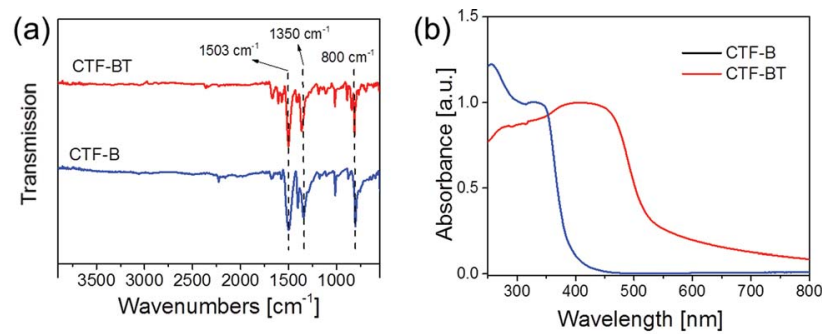

(c)

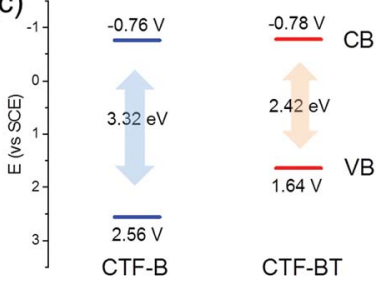

(d)

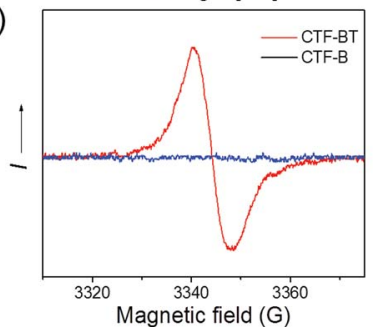

Fig. 2 (a) FT-IR spectra, (b) UV-vis DR spectra, (c) VB and CB positions and (d) electron paramagnetic resonance (EPR) spectra of CTF-BT and CTF-B.

To reveal the electronic properties of the CTFs, cyclic voltammetry (CV) measurements were conducted (Fig. S14, ESI $\dagger$ ). Both CTFs exhibited similar CB positions with $-0.78 \mathrm{~V}$ for CTF-BT and $-0.76 \mathrm{~V}$ for CTF-B (Fig. 2c). The valence bands (VB) positions of the polymers were derived by extracting the $\mathrm{CB}$ values from the optical band gaps, revealing VBs of $1.64 \mathrm{~V}$ for CTF-BT and $2.56 \mathrm{~V}$ for CTF-B (Fig. 2c). Electron paramagnetic resonance (EPR) spectra showed a clearly enhanced signal for CTF-BT under visible light irradiation (Fig. 2d), indicating the photogeneration of paramagnetic species (radical or conduction band electrons) in the polymer network. ${ }^{28}$ No apparent EPR signal was observed for CTF-B in either the dark or when irradiated by visible light, which was likely caused by its narrow absorption in the visible region.

The photocatalytic reduction of 4-nitrophenol (4-NP) to 4-aminophenol (4-AP), which is usually catalyzed by gold-containing catalysts, ${ }^{29-31}$ was chosen as a simple model reaction to evaluate the photocatalytic activity of the CTFs. Using CTF-BT containing the ordered hollow structure as photocatalyst, the reduction of 4-NP was completed after $50 \mathrm{~min}$ with an average turnover frequency (TOF) of $0.18 \mathrm{~h}^{-1}$ (Fig. 3a). No 4-AP was detected in the absence of CTF-BT or light irradiation, indicating their indispensable role for the catalytic reaction. A bulk CTF-BT without using $\mathrm{SiO}_{2}$ NPs as template was also used. It was shown that the bulk CTF-BT without the uniformed hollow structure only achieved a conversion of $46 \%$ with a TOF of 0.08 $\mathrm{h}^{-1}$, despite the same chemical composition and similar BET surface area. The superior photocatalytic activity of CTF-BT with the hollow structure could be likely attributed to two main factors: (i) the enhanced mass transfer throughout the interconnected hollow polymer network and (ii) the enhanced light absorption via the multiple light reflections inside the polymer (Fig. S15d, ESI $\dagger$ ). ${ }^{23,32}$ A control experiment using fine-ground CTF-BT as photocatalyst, wherein the hollow structure was destroyed, led only to a reduced conversion of $c a$. $75 \%$ of 4 -AP.
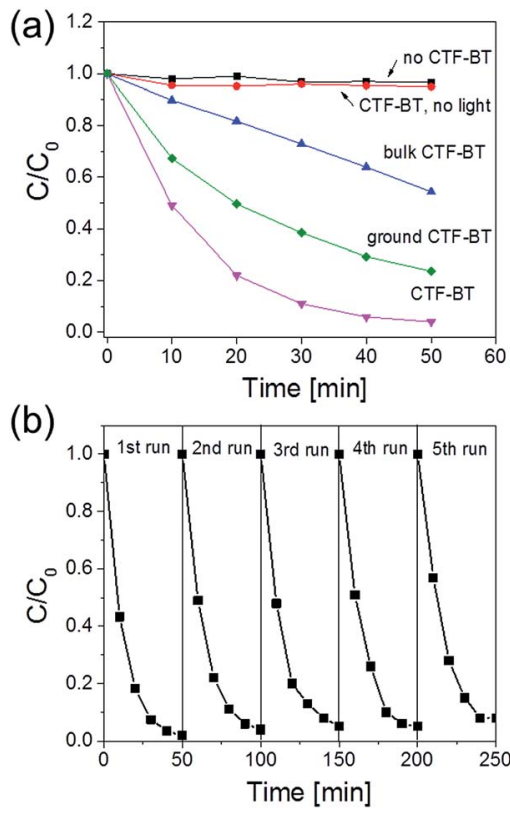

Fig. 3 (a) Photoreduction rates of 4-NP to 4-AP using hollow CTF-BT as photocatalyst with control experiments. Reaction conditions: CTFBT (5 mg), 2 mM 4-NP (1 ml), $\mathrm{NaBH}_{4}(10 \mathrm{mg}), 1 \mathrm{M} \mathrm{NaOH}(10 \mu \mathrm{l}), \mathrm{H}_{2} \mathrm{O} /$ EtOH $(3: 1) 4 \mathrm{ml}$, white LED lamp $(\lambda>420 \mathrm{~nm})$. (b) Repeating experiments of photoreduction of 4-NP.

The fluorescence decay measurements showed similar lifetimes of photogenerated charge carriers of the hollow CTF-BT, bulk CTF-BT, as well as the ground one, suggesting that the different photocatalytic efficiencies rather originated from the morphology. To note, CTF-B did not achieve any photocatalytic activity due to its weak absorption in the visible region (Fig. S18, ESI $\dagger$ ).

To gain more insights of the reaction mechanism and the photogenerated electron transfer between the photocatalyst and substrate during the catalytic process, we first carried out photoluminescence quenching experiments. It could be observed that the emission intensity of CTF-BT gradually diminished with increasing concentration of 4-NP, indicating an electron transfer between CTF-BT in its excited state and 4-NP (Fig. S19, ESI $\dagger$ ), leading to the formation of an "activated" anionic radical intermediate of 4-NP. The following step of the catalytic cycle was then investigated by verifying the role of $\mathrm{NaBH}_{4}$ in the photocatalytic process. According to the literature, borohydride could function not only as hydrogen source, but also as electron donor. ${ }^{33}$ By replacing $\mathrm{NaBH}_{4}$ with a hydrogenpoor agent, sodium tetraphenylborate (NaTPB), which only acted as an electron donor, the reduction of 4-NP was still successful (Fig. S20, ESI $\dagger$ ). This indicates that an extra hydrogen-donating reagent such as $\mathrm{NaBH}_{4}$ was not mandatory for the photoreduction of 4-NP. The hydrogen species could also originate from the protic solvents, i.e., water. This could be confirmed by using aprotic solvent dimethyl sulfoxide (DMSO), where no apparent reduction of 4-NP was determined (Fig. S20, ESI $\dagger$ ). Based on those observations, a reaction mechanism could be proposed, as illustrated in Fig. S21. $\dagger$ Upon visible light 
irradiation, the electron donor, $\mathrm{NaBH}_{4}$ or NaTPB, was oxidized by the photogenerated hole in CTF-BT, followed by the electron transfer from the CB of CTF-BT onto 4-NP to form its activated anionic radical intermediate, which simultaneously extracted hydrogen species either from an extra hydrogen source $\left(\mathrm{NaBH}_{4}\right)$ or the protic solvent (water), resulting in the final product 4-AP.

Repeating experiments revealed that CTF-BT could be used for five extra cycles without significantly losing its catalytic efficiency (Fig. 3b). No clear change in the FTIR spectrum or the SEM image was observed (Fig. S22 and S23, ESI $\dagger$ ), demonstrating the high stability of both texture and chemical structure of the polymer.

\section{Conclusions}

In conclusion, we presented a new facile method to construct nanoporous hollow triazine-based polymer frameworks via $\mathrm{TfOH}$ vapor-assisted solid phase synthesis of aromatic nitriles with using silica templates under mild reaction conditions. Stable CTFs with unique interconnected pores with diameters of $c a$. $300 \mathrm{~nm}$ could be obtained without undesired side reactions, such as decomposition or carbonization, or metal catalyst residue compared to common and metal-catalyzed triazine network formations under high temperature. Via variation of the nitrilefunctionalized organic semiconductor units, polymer networks with tuneable optical and electronic properties could be synthesized. The enhanced photocatalytic activity of the nanoporous polytriazine networks was demonstrated in the photoreduction reaction of 4-NP to 4-AP due to more efficient mass transfer and light absorption. We believe this metal catalyst-free solid phase synthetic method at lower temperature could provide a promising platform of designing triazine-based polymer networks with defined optoelectronic properties and morphologic structures for a broader application possibility in photocatalysis.

\section{Acknowledgements}

The authors thank the Max Planck Society for the financial support. W. H. thanks the China Scholarship Council (CSC) (No. 201406240010) for the scholarship. Z. J. W. thanks the fellowship by the Excellence Initiative (DFG/GSC 266) of the graduate school of excellence "MAINZ" (Materials Science in Mainz). B. C. M. acknowledges the financial support from DAAD, CAPES and CNPq. D. G thanks the Fonds der Chemischen Industrie (FCI) for the Kekule scholarship.

\section{Notes and references}

1 P. Kuhn, M. Antonietti and A. Thomas, Angew. Chem., Int. Ed., 2008, 47, 3450-3453.

2 K. Sakaushi, E. Hosono, G. Nickerl, T. Gemming, H. Zhou, S. Kaskel and J. Eckert, Nat. Commun., 2013, 4, 1485.

3 L. Hao, J. Ning, B. Luo, B. Wang, Y. Zhang, Z. Tang, J. Yang, A. Thomas and L. Zhi, J. Am. Chem. Soc., 2014, 137, 219-225. 4 K. A. See, S. Hug, K. Schwinghammer, M. A. Lumley, Y. Zheng, J. M. Nolt, G. D. Stucky, F. Wudl, B. V. Lotsch and R. Seshadri, Chem. Mater., 2015, 27, 3821-3829.
5 S. N. Talapaneni, T. H. Hwang, S. H. Je, O. Buyukcakir, J. W. Choi and A. Coskun, Angew. Chem., Int. Ed., 2016, 55, 3106-3111.

6 R. Palkovits, M. Antonietti, P. Kuhn, A. Thomas and F. Schüth, Angew. Chem., Int. Ed., 2009, 48, 6909-6912.

7 C. E. Chan-Thaw, A. Villa, P. Katekomol, D. Su, A. Thomas and L. Prati, Nano Lett., 2010, 10, 537-541.

8 K. Kamiya, R. Kamai, K. Hashimoto and S. Nakanishi, Nat. Commun., 2014, 5, 5040.

9 C. E. Chan-Thaw, A. Villa, D. Wang, V. D. Santo, A. Orbelli Biroli, G. M. Veith, A. Thomas and L. Prati, ChemCatChem, 2015, 7, 2149-2154.

10 X. Zhu, C. Tian, S. M. Mahurin, S.-H. Chai, C. Wang, S. Brown, G. M. Veith, H. Luo, H. Liu and S. Dai, J. Am. Chem. Soc., 2012, 134, 10478-10484.

11 A. Bhunia, I. Boldog, A. Möller and C. Janiak, J. Mater. Chem. A, 2013, 1, 14990-14999.

12 M. Saleh, S. B. Baek, H. M. Lee and K. S. Kim, J. Phys. Chem. C, 2015, 119, 5395-5402.

13 S. Hug, M. B. Mesch, H. Oh, N. Popp, M. Hirscher, J. Senker and B. V. Lotsch, J. Mater. Chem. A, 2014, 2, 5928-5936.

14 R. S. Sprick, J.-X. Jiang, B. Bonillo, S. Ren, T. Ratvijitvech, P. Guiglion, M. A. Zwijnenburg, D. J. Adams and A. I. Cooper, J. Am. Chem. Soc., 2015, 137, 3265-3270.

15 C. Butchosa, T. O. McDonald, A. I. Cooper, D. J. Adams and M. A. Zwijnenburg, J. Phys. Chem. C, 2014, 118, 4314-4324.

16 S. Ren, M. J. Bojdys, R. Dawson, A. Laybourn, Y. Z. Khimyak, D. J. Adams and A. I. Cooper, Adv. Mater., 2012, 24, 23572361.

17 K. Sakaushi and M. Antonietti, Acc. Chem. Res., 2015, 48, 1591-1600.

18 F. Niu, L. Tao, Y. Deng, H. Gao, J. Liu and W. Song, New J. Chem., 2014, 38, 5695-5699.

19 K. Schwinghammer, S. Hug, M. B. Mesch, J. Senker and B. V. Lotsch, Energy Environ. Sci., 2015, 8, 3345-3353.

20 J. Sun, J. Zhang, M. Zhang, M. Antonietti, X. Fu and X. Wang, Nat. Commun., 2012, 1139.

21 C. T. Dinh, H. Yen, F. Kleitz and T. O. Do, Angew. Chem., Int. Ed., 2014, 53, 6618-6623.

22 W.-N. Wang, W.-J. An, B. Ramalingam, S. Mukherjee, D. M. Niedzwiedzki, S. Gangopadhyay and P. Biswas, J. Am. Chem. Soc., 2012, 134, 11276-11281.

23 C. C. Nguyen, N. N. Vu and T.-O. Do, J. Mater. Chem. A, 2015, 3, 18345-18359.

24 A. Ajayaghosh, Chem. Soc. Rev., 2003, 32, 181-191.

25 J. Bi, W. Fang, L. Li, J. Wang, S. Liang, Y. He, M. Liu and L. Wu, Macromol. Rapid Commun., 2015, 36, 1799-1805.

26 C. P. Wilgus, S. Downing, E. Molitor, S. Bains, R. M. Pagni and G. W. Kabalka, Tetrahedron Lett., 1995, 36, 3469-3472.

27 R. Li, Z. J. Wang, L. Wang, B. C. Ma, S. Ghasimi, H. Lu, K. Landfester and K. A. I. Zhang, ACS Catal., 2016, 6, 11131121.

28 D. Hollmann, M. Karnahl, S. Tschierlei, K. Kailasam, M. Schneider, J. R. Radnik, K. Grabow, U. Bentrup, H. Junge and M. Beller, Chem. Mater., 2014, 26, 1727-1733.

29 J. He, W. Ji, L. Yao, Y. Wang, B. Khezri, R. D. Webster and H. Chen, Adv. Mater., 2014, 26, 4151-4155. 
30 J. Li, C.-y. Liu and Y. Liu, J. Mater. Chem., 2012, 22, 84268430.

31 X.-H. Li, X. Wang and M. Antonietti, Chem. Sci., 2012, 3, 2170-2174.
32 Y. S. Jun, E. Z. Lee, X. Wang, W. H. Hong, G. D. Stucky and A. Thomas, Adv. Funct. Mater., 2013, 23, 3661-3667.

33 M. Kropp and G. B. Schuster, Tetrahedron Lett., 1987, 28, 5295-5298. 